\title{
DISCLAIMER
}

This report was prepared as an account of work sponsored by an agency of the United States Government. Neither the United States Government nor any agency thereof, nor any of their employees, makes any warranty, express or implied, or assumes any legal liability or responsibility for the accuracy, completeness, or usefulness of any information, apparatus, product, or process disclosed, or represents that its use would not infringe privately owned rights. Reference herein to any specific commercial product, process, or service by trade name, trademark, manufacturer, or otherwise does not necessarily constitute or imply its endorsement, recommendation, or favoring by the United States Government or any agency thereof. The views and opinions of authors expressed herein do not necessarily state or reflect those of the United States Government or any agency thereof.

SLAC-PUB-7508 May 1997

$$
\text { CONF-970503-184 }
$$

\section{Beam-Beam Simulations with Non-Gaussian Distributions for SLC and SLC-2000*}

\author{
K.L.F. Bane, P. Chen, F. Zimmermann \\ Stanford Linear Accelerator Center \\ Stanford University, Stanford, CA 94309, USA
}

\section{RECEIVED \\ JUN 269997 \\ O.STI}

Due to various upstream beam manipulations, the longitudinal bunch shape at the interaction point of the Stanford Linear Collider (SLC) is highly non-Gaussian. In this paper, we report beam-beam simulations with realistic longitudinal bunch shapes for the present SLC parameters and for the SLC-2000 luminosity upgrade. The simulation results allow us to estimate the luminosity enhancement due to the pinch effect and to find optimum parameter settings for the bunch compressor and the linac.

Presented at the 1997 Particle Accelerator Conference (PAC97), Vancouver, B.C., Canada, May 12-16, 1997

${ }^{*}$ Work supported by the U.S. Department of Energy contract DE-AC03-76SF00515. 


\section{DISCLAIMER}

Portions of this document may be illegible in electronic image products. Images are produced from the best available original document. 


\title{
BEAM-BEAM SIMULATIONS WITH NON-GAUSSIAN DISTRIBUTIONS FOR SLC AND SLC-2000*
}

\author{
K.L.F. Bane, P. Chen, F. Zimmermann \\ Stanford Linear Accelerator Center, Stanford University, CA 94309, USA
}

\begin{abstract}
Due to various upstream beam manipulations, the longitudinal bunch shape at the interaction point of the Stanford Linear Collider (SLC) is highly non-Gaussian. In this paper, we report beam-beam simulations with realistic longitudinal bunch shapes for the present SLC parameters and for the SLC-2000 luminosity upgrade. The simulation results allow us to estimate the luminosity enhancement due to the pinch effect and to find optimum parameter settings for the bunch compressor and the linac.
\end{abstract}

\section{INTRODUCTION}

Many linear-collider beam-beam simulations performed in the past have assumed simplified longitudinal beam distributions at the interaction point, usually a Gaussian and, sometimes, a uniform distribution. However, real bunch distributions are neither Gaussian nor uniform.

At the Stanford Linear Collider, the first and only linear collider in the world, the longitudinal distribution at the interaction point (IP) is clearly non-Gaussian. The longitudinal bunch shape at the SLC IP is determined by a variety of effects [1]: bunch-lengthening in the damping rings, (over)compression, If curvature and second-order momentum compaction in the ring-to-linac transfer lines, if curvature and longitudinal wake fields in the linac, and, finally, another bunch compression or expansion plus synchrotron radiation in the collider arcs. As a result of all these effects, the bunch distribution at the interaction point (IP) differs markedly from the idealized distributions usually considered. There is, in fact, some evidence that luminosity predictions derived from Gaussian-bunch simulations compare poorly with SLC observations [2].

A study of non-Gaussian beam-beam collisions is further motivated by the proposed luminosity upgrade SLC-2000 [3], for which, due to stronger bunch-length compression in the arcs (necessary to reduce the hourglass effect for the smaller $\beta_{y}^{\star}$ ), the distortion of the IP distribution will be even more pronounced.

In this paper, we report recent simulation results of beam-beam collisions, both for the present SLC and for the SLC-2000 upgrade. The simulations are based on realistic IP bunch distributions including all the effects in longitudinal phase space mentioned above, and starting with the potential-well distorted beams in the damping rings. The purpose of these simulations is: (1) to provide an estimate of the expected luminosity and of the pinch effect for real,

\footnotetext{
* Work supported by the U.S. Department of Energy under contract DE-AC03-76SF00515.
}

\begin{tabular}{|l|c|c|}
\hline parameter & SLC-1996 & SLC-2000 \\
\hline \hline$\gamma \epsilon_{x}(\mathrm{~m})$ & $5.9 \times 10^{-5}$ & $4.0 \times 10^{-5}$ \\
$\gamma \epsilon_{y}(\mathrm{~m})$ & $1.9 \times 10^{-5}$ & $1.1 \times 10^{-5}$ \\
$\theta_{x}^{\star}$ & $350 \mu \mathrm{rad}$ & $550 \mu \mathrm{rad}$ \\
$\theta_{y}^{\star}$ & $350 \mu \mathrm{rad}$ & $500 \mu \mathrm{rad}$ \\
$\beta_{x}^{\star}$ & $5.4 \mathrm{~mm}$ & $1.47 \mathrm{~mm}$ \\
$\beta_{y}^{\star}$ & $1.7 \mathrm{~mm}$ & $0.5 \mathrm{~mm}$ \\
$\delta_{r m s}$ & $0.1 \%$ & $0.2 \%$ \\
$N$ & $3.5 \times 10^{10}$ & $3.5 \times 10^{10}$ \\
$\sigma_{x}^{\star}$ & $1.9 \mu \mathrm{m}$ & $810 \mathrm{~nm}$ \\
$\sigma_{y}^{\star}$ & $600 \mathrm{~nm}$ & $250 \mathrm{~nm}$ \\
\hline
\end{tabular}

Table 1: Nominal IP beam parameters for the 1996 SLC and for the SLC-2000 upgrade.

non-Gaussian bunches, (2) to determine settings of compressor voltage and linac phase which are optimized for maximum luminosity, and (3) to provide guidance for the operation of two newly installed IP bunch-length monitors $[7,8]$. Table 1 lists nominal IP beam parameters for the present SLC and for the SLC-2000 upgrade, which will be assumed in the remainder of this paper.

\section{OPTIMUM BUNCH SHAPE FOR THE SLC}

The longitudinal bunch distribution at the SLC IP is calculated with the program LITRACK [4], by which we model the longitudinal dynamics in the damping rings and in all downstream systems. This distribution is used as an input to the beam-beam program Guinea-pig [5]. Other input parameters, including the angular divergence and the spot size, are taken from Table 1. We have performed a series of simulations to study the dependence of the IP bunch shape and luminosity on the linac phase and bunch compressor voltage.

Figure 1 illustrates how a change of the average linac phase $\phi$ affects the IP bunch shape. Displayed is the longitudinal beam distribution for three different linac phases, with the center figure representing the nominal case. In this report, the phase $\phi$ is measured with respect to the crest of the linac If, with $\phi<0$ (or $z<0$ ) to the front of the bunch.

In Fig. 2, the IP bunch length, energy spread and luminosity enhancement factor are shown as a function of the linac phase, for a constant compressor voltage $V_{c}$. The luminosity enhancement factor $H_{D}$ is defined as the ratio of the effective luminosity $L$ and the geometric luminosity $L_{0}$, where the latter is calculated from the IP beam size without collision. The enhancement factor can be larger than 1 due to the pinch effect (mutual focusing of the two beams by each other) or smaller than 1 due to the hourglass ef- 
fect (i.e., the angular divergence of the two beams). During SLC operation, the injection time is continually adjusted in order to compensate for diurnal drifts of the linac-rf reference phases. Usually, this adjustment is done by minimizing the beam energy spread at the end of the linac. This is not necessarily the best choice in regard to the pinch effect, although Figs. 2 (a) and (d) suggest that it is not too far from the optimum. For comparison, the luminosity enhancement for a Gaussian bunch, one with the same rms length, is also shown in Fig. 2 (a), by the dotted line. In this particular case, the enhancement factors for Gaussian and real distributions are almost identical.
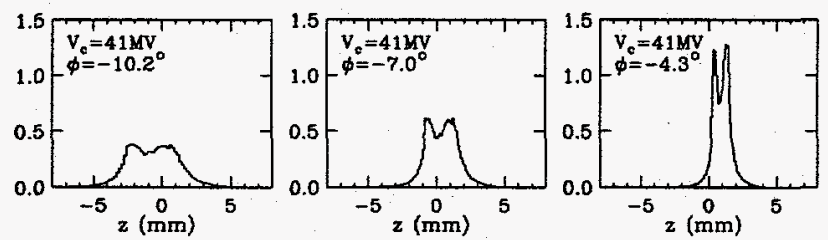

Figure 1: IP bunch shape for different linac phases. The vertical axis gives the instantaneous current in units of $k A$.
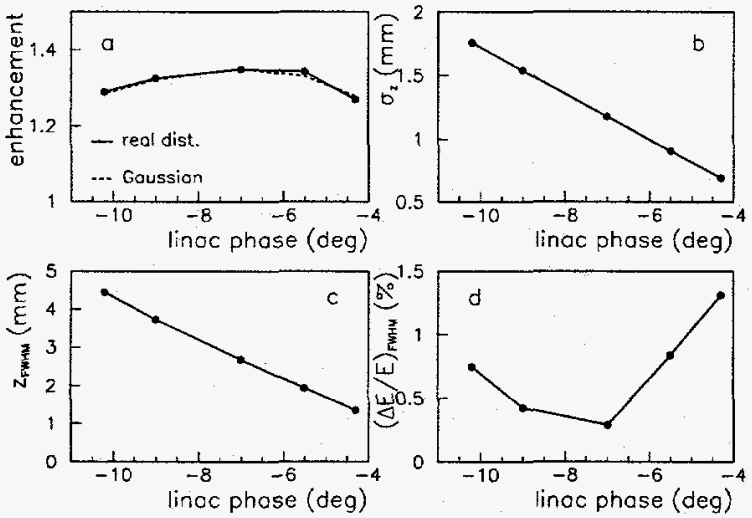

Figure 2: Luminosity enhancement (a), rms bunch length (b), FWHM bunch length (c) and FWHM energy spread (d) as a function of the linac phase, for the 1996 parameters.

The second parameter that can substantially affect the IP distribution is the voltage of the compressor rf. Examples of IP distributions for different compressor voltages are depicted in Fig. 3, where in each case the linac phase is adjusted to minimize the final energy spread. Figure 3 shows that a too small compressor voltage may result in a very spiky distribution, which is unlikely to show a large pinch effect. The nominal if voltage is $41 \mathrm{MV}$ (center case of Fig. 3), which yields an rms bunch length in the linac of about $1.2 \mathrm{~mm}$. Figure 4 displays simulation results which illustrate how the IP bunch length, the energy spread and the luminosity vary with the compressor voltage, again minimizing the energy spread. For comparison, the dotted line in Fig. 4 (a) shows the luminosity enhancement for a Gaussian distribution of equal rms length. The clear differ- ence between the dotted and solid lines demonstrates that a Gaussian is not always a good approximation.

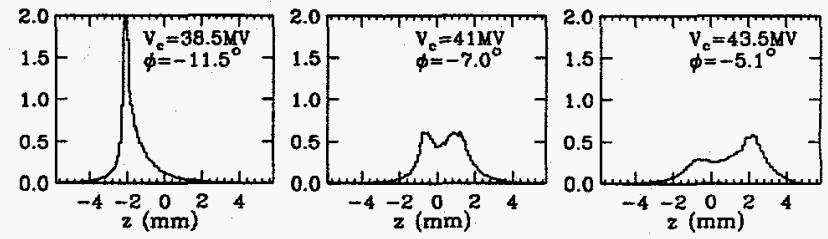

Figure 3: IP bunch shape for different compressor voltages. The vertical axis gives the instantaneous current in units of kA.
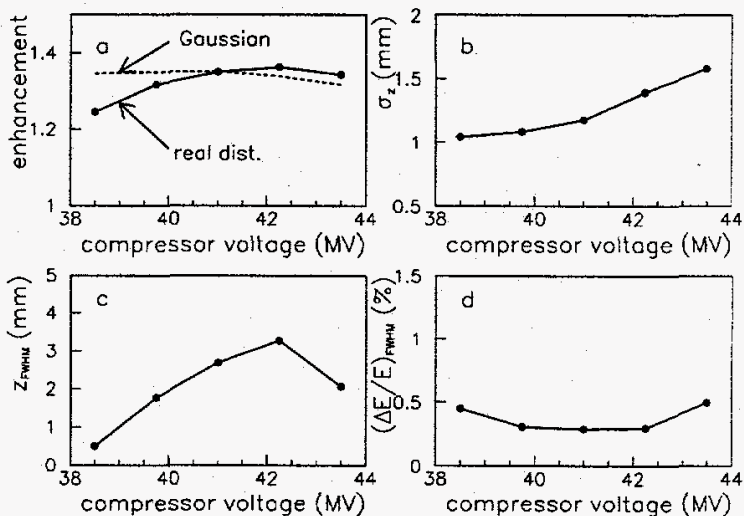

Figure 4: Luminosity enhancement (a), rms bunch length (b), FWHM bunch length (c) and FWHM energy spread (d) as a function of the compressor voltage for the 1996 parameters.

\section{BUNCH LENGTH MONITORING}

To optimize the luminosity enhancement, for the 1997 SLC run two novel bunch-length monitors have been installed in the SLC final focus: 1) a laser-heterodyne monitor based on the laser-wire system [8], which can be used to measure the entire Fourier transform of the beam profile; 2) a broadband rf monitor, which detects signals in 4 different frequency ranges $(8-11.6 \mathrm{GHz}, 11.6-19 \mathrm{GHz}, 19-110 \mathrm{GHz}, 59-110$ $\mathrm{GHz}$ ) and which will provide continuous information about the bunch length. Figure 5 displays the expected signals in the four channels of the rf monitor for various linac phases and compressor voltages equivalent to those considered in Figs. 2 and 4. The signals are calculated by performing a fast fourier transform of the bunch distribution and then integrating over the different frequency ranges. As expected, channel 4 is the most sensitive to the bunch length. In the $1997 / 98$ SLC run, the signal of this channel will allow us to constantly monitor the IP bunch length of electron and positron beams, and, thus, to ascertain that the linac and compressor are set up properly for maximum pinch effect. 

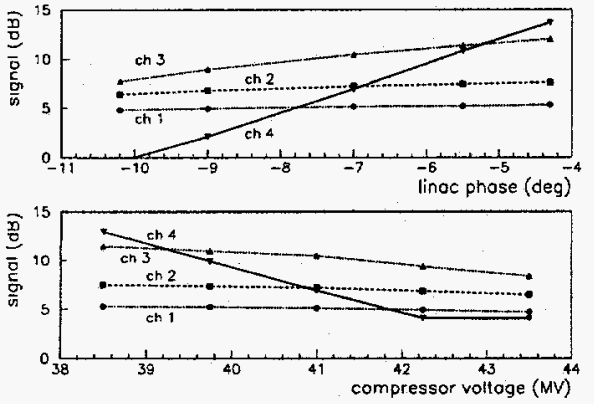

Figure 5: Predicted signal in the 4 channels of the newly installed RF bunch-length monitor [7], as a function of the linac phase and the compressor voltage (same horizontal scale as in Figs. 2 and 4, respectively).

\section{LUMINOSITY ENHANCEMENT AT SLC-2000}

In simulation studies for SLC-2000, we have used the parameters listed in the right-hand column of Table 1 , and we have considered four different values of the compressor if voltage. For each compressor voltage, we have varied the linac phase $\phi$ within a certain range constrained by the resulting energy spread (see Fig. 6). Given the present SLC2000 final-focus energy acceptance of $0.6 \%$ [3], the optimal compressor voltage is $V_{c} \approx 40 \mathrm{MV}$ and the luminosity enhancement is $20-30 \%$. However, if the energy acceptance can be increased to $1.4 \%$, a shorter IP bunch length and an enhancement up to $60 \%$ would become possible, with a compressor voltage $V_{c}=41 \mathrm{MV}$. Our simulation results for the 1996 SLC and SLC-2000 are summarized in Table 2.
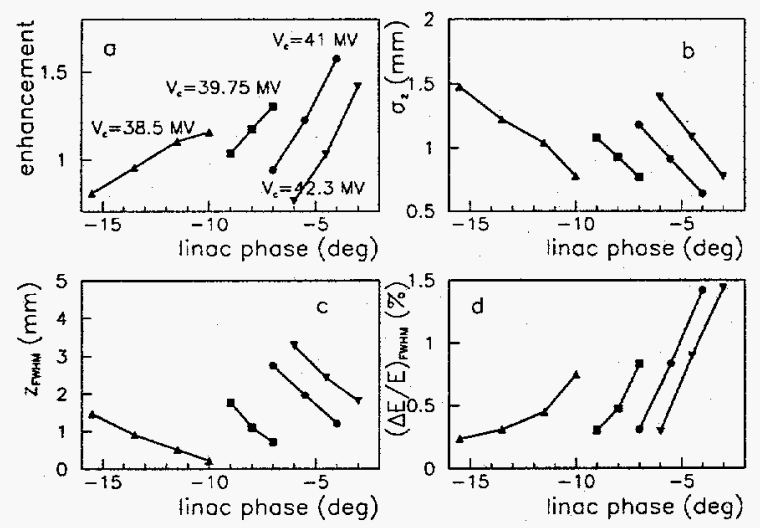

Figure 6: Luminosity enhancement (a), rms bunch length (b), FWHM bunch length (c) and FWHM energy spread (d), as a function of the linac phase for SLC-2000, considering four different compressor voltages: $38.5 \mathrm{MV}$ (triangles), $39.75 \mathrm{MV}$ (squares), $41 \mathrm{MV}$ (rectangles), and 42.25 MV (inverse triangles).

\begin{tabular}{|l|c|c|}
\hline parameter & SLC-1996 & SLC-2000 \\
\hline \hline$\phi$ & $-7^{\circ}$ & $-4^{\circ}$ \\
$V_{c}$ & $41 \mathrm{MV}$ & $41 \mathrm{MV}$ \\
$\sigma_{z}$ & $1.18(1.0) \mathrm{mm}$ & $0.64(0.5) \mathrm{mm}$ \\
\hline$L_{0}$ & $110 \mathrm{Zs} / \mathrm{hr}$ & $640 \mathrm{Zs} / \mathrm{hr}$ \\
$L$ & $150(149) \mathrm{Zs} / \mathrm{hr}$ & $998(1073) \mathrm{Zs} / \mathrm{hr}$ \\
$H_{D}$ & $1.35(1.34)$ & $1.56(1.68)$ \\
\hline$\theta_{x}$ & $0.62(0.62) \mathrm{mrad}$ & $1.18(1.29) \mathrm{mrad}$ \\
$\theta_{y}$ & $509(532) \mu \mathrm{rad}$ & $616(661) \mu \mathrm{rad}$ \\
$\left\langle\frac{d E}{E}\right\rangle$ & $0.039(0.042) \%$ & $0.53(0.61) \%$ \\
$\sigma_{E}$ & $49(54) \mathrm{MeV}$ & $441(482) \mathrm{MeV}$ \\
\hline$N_{\gamma} /$ electron & $0.68(0.68)$ & $1.77(1.80)$ \\
$\left\langle E_{\gamma}\right\rangle$ & $25(26) \mathrm{MeV}$ & $141(154) \mathrm{MeV}$ \\
\hline
\end{tabular}

Table 2: Predicted luminosity, spent-beam and photonbeam characteristics for the 1996 SLC and SLC-2000, obtained in beam-beam simulations with realistic distributions using the program Guinea-pig [5]. The symbol $L_{0}(L)$ denotes the luminosity without (with) pinch and hourglass effect, and $H_{D}$ is the enhancement factor $L / L_{0}$, which, in the simulation, was optimized by adjusting the linac phase for a constant compressor voltage of $41 \mathrm{MV}$. The values given in parentheses are results for a Gaussian distribution (see also [6]). The last two rows refer to the beamstrahlung photons.

\section{CONCLUSION}

Using realistic longitudinal bunch distributions, we have determined the optimum compressor setting and the optimum linac phase for the 1996 SLC and for the SLC2000 upgrade. We find that, in most cases, the Gaussian approximation to the bunch shape gives nearly the same luminosity enhancement factor as the actual bunch shape. For the 1996 SLC parameters, the maximum enhancement due to the pinch effect is about $30 \%$, while for SLC- 2000 an enhancement of $20-30 \%$ is expected for the present final-focus design optics, which may be increased to $60 \%$ with an improved final-focus energy bandwidth.

\section{Acknowledgement}

We thank $O$. Napoly for stimulating discussions.

\section{REFERENCES}

[1] K.L.F. Bane, SLAC/AP-80 (1990).

[2] T. Markiewicz, private communication (1997).

[3] M. Breidenbach et al., "SLC-2000: A luminosity upgrade for the SLC", LINAC 96, and SLAC-PUB-7268 (1996).

[4] K. Bane, unpublished.

[5] D. Schulte, private communication (1997).

[6] O. Napoly, SLAC CN-408 (1996).

[7] F. Zimmermann, G. Yocky, et al., "An RF bunch-length monitor for the SLC final focus", these proceedings (1997).

[8] T. Kotseroglou et al., "A laser-heterodyne bunch-length monitor for the SLC interaction point", these proceedings (1997). 